\title{
Sensing schedule optimization to minimize interference with primary users in cognitive radio network
}

\author{
Suddhendu DasMahapatra ${ }^{1}$, Ishita Gandhi ${ }^{2}$, Kartik Nair ${ }^{3}$, Shivendra Nath Sharan ${ }^{4}$ \\ ${ }^{1,2}$ Manipal University Jaipur, Rajasthan, India \\ ${ }^{3}$ Virginia Polytechnic Institute and State University, USA \\ ${ }^{4}$ NIIT University, Rajasthan, India
}

\begin{tabular}{l}
\hline Article Info \\
\hline Article history: \\
Received Jul 26, 2019 \\
Revised Sep 1, 2019 \\
Accepted Oct 25, 2019 \\
\hline
\end{tabular}

\section{Keywords:}

Cognitive radio

Genetic algorithm

Sensing scheduling

Spectrum sensing

\begin{abstract}
Radio Frequency (RF) spectrum, which is a limited resource, is facing the challenge of fulfilling the need of ever-growing users. To accommodate these users in an efficient manner, the idea of Cognitive Radio (CR) was proposed. It allows unlicensed users to use the licensed bands when the primary user is not using its band. To check the availability of the free spectrum, the system should be able to sense the RF environment around it, thus spectrum sensing becomes an important aspect of CR. In this work, the Genetic Algorithm (GA) is used to determine the periodic sensing schedule to maximize the number of transmission opportunities for unlicensed band users as well as to minimize interference with licensed users. This optimization scheme generates a maximum number of transmission opportunities with minimum interruption to the licensed user's communication. The MATLAB software is used for simulation.
\end{abstract}

Copyright $\odot 2020$ Institute of Advanced Engineering and Science. All rights reserved.

\section{Corresponding Author:}

Suddhendu DasMahapatra, Manipal University Jaipur, Rajasthan, India.

Email: suddm.kgp@gmail.com

\section{INTRODUCTION}

The radio spectrum space is a hypothetical space occupied by the radio signals. This hyperspace can be represented as a frequency spectrum and is further divided into several frequency bands. The telecommunication regulatory authorities, then, divided the entire frequency spectrum into two categories- licensed bands and unlicensed bands. The licensed bands are those frequencies that are allocated to specific users and are not freely available to any other user. While the other bands that remain freely available for the users are known as the unlicensed bands.

The unlicensed bands are crucial as the majority of communication systems make use of these frequencies. As it is freely available to all the users, it addresses the problem of the digital divide. It provides marginalized communities with a low-cost solution to the problems concerning access to information. It provides the platform for the development of new technologies and benefits new start-ups to make use of these frequencies and also helps to expand their services and market as it doesn't involve any costly investment for buying new licensed frequency bands. As the number of users is increasing by leaps and bounds, the idea of running out of frequency has become a common scare.

The need for wireless communication has increased tremendously in the past few decades [1], which also means that the number of users in the frequency spectrum has increased by many folds. With the increasing demand for the spectrum, the management of the spectrum becomes of most importance. Presently, much multiple access and multiplexing schemes are adopted to allow multiple users to avail the same frequency band simultaneously and thus ensuring optimum usage of frequency bands by the designated users of that band. 
However, the idea of CR [2-3] goes a step further. It intends at making use of any spectrum hole available throughout the frequency spectrum. A spectrum hole is that frequency band which is currently not being used by its Primary User (PU) i.e. licensed user and is idle. These spectrum holes [4] can be present in any of the available frequency bands and if these holes used will lead to a more efficient frequency usage. The CR allows the unlicensed users i.e. Secondary Users (SUs) to make use of these spectrum holes while the primary user is not using it, but the SUs must vacate this frequency as soon as the PU resumes its communication [5]. The PU always has the first right to make use of the licensed band and the SU must make sure that the SU's communication does not interfere with the PU communication or affect the performance of the PU communication [6]. Therefore, spectrum sensing becomes an important aspect of the implementation of any CR network. CR needs to sense the spectrum regularly to detect the spectrum holes. However, the spectrum sensing faces its challenges such as keeping sensing cost under a constraint and at the same time presenting the CR with a maximum number of opportunities [7]. Therefore, it is necessary to find out the optimum period of the sensing event by SU so that it may produce an acceptable performance and at the same time adheres to the defined constraints. The larger the number of sensing in a given time slot, the more it enhances the sensing performance i.e. less interference with PU. But larger sensing time, less is the time for data transmission. This may decrease the overall throughput of the SU. Thus an optimum sensing scheduling is essential for a CR network. In this work, the optimization has been carried out using GA, which is an evolutionary optimization technique that is based on the idea of natural evolution. In recent times, GA has gathered much attention and found a widespread application.

\section{RELATED WORK}

The static spectrum allocation has a widespread application in wireless communication where the licensed spectrum band is allocated to a specific user alone. The allocation of the spectrum cannot be changed and only the designated user communicates using the licensed band. It facilitates the system design but compromises the efficiency of the channels whereas the concept of dynamic spectrum allocation enables us to overcome the drawbacks of the static allocation [5]. It is observed that the dynamic allocation of spectrum has

CR can be viewed as further advancement in the dynamic allocation of the channel [8]. It was proposed to address the issue of spectrum efficiency and has been receiving increasing attention in recent years. There have been many significant developments in the past few years on cognitive radios. Presently, much stress is being laid on the importance of the need for efficient spectrum sensing [9].

Spectrum sensing is carried out to find the spectrum holes and provide high-resolution capabilities. While sensing the spectrum, the CR also makes sure that it does not interfere with the PU's communication. The SUs must vacate the frequency immediately as the PU resumes its communication and should not interfere or affect the quality of service entitled to the primary user. To avoid interference, it is needed to optimize the sensing period. The authors of [7] addressed this problem. The optimal sensing period to minimize the transmission delay is proposed by [10]. Authors of [11-12] proposed a scheme based on GA to choose the transmission parameters of the CR network. But they had not taken the sensing period and sensing schedule into consideration. The authors of [13] examined the effectiveness of GA in power distribution in a sharing-based model of CR. Joint optimization of power and subcarrier distribution for orthogonal frequency division multiplexing based CR system is explored in [14]. In [15-16], the authors maximized the overall throughput of the CR network using evolutionary game theory. In [17], the authors used a combination of enhanced spectrum sensing (ESS) and hybrid spectrum sharing (HSS) to improve the spectral efficiency of SUs. In another work, authors of [18] examined the resource sharing problem in OFDM based CR relay network with multiple relays to maximize the transmission rate. Accurate identification of the modulation scheme used to modulate PU signal is explored in [19]. Authors of [20] projected a preventive multi-channel access model for prioritized CR networks using queuing theory to resolve the spectrum scarcity issue. The sensing duration is altered dynamically, with reference to changing RF environment, to maximize overall throughput of a CR, is presented in [21]. Zhang and Xie [22-23] demonstrated a neural network-based CR engine that can learn both fixed and variable aspects. Zhao et al. [24] proposed a CR device based on Ant Colony Optimization (ACO). The impact of several transmission parameters on a GA based CR is studied in [25].

\section{SYSTEM MODEL}

A theoretical model of a CR network is shown in Figure 1. To detect the presence of PU in the RF environment, SUs need to sense the spectrum periodically. The power consumption for sensing and time required to sense the spectrum may be considered as sensing cost. It is desired that the sensing cost should 
not be large. However, sensing outcome often depends on the sensing timing and sensing period. As shown in Figure 2, sensing period and sensing time are the two main factors to determine sensing outcome, which is considered as the time available for data transmission for SUs.

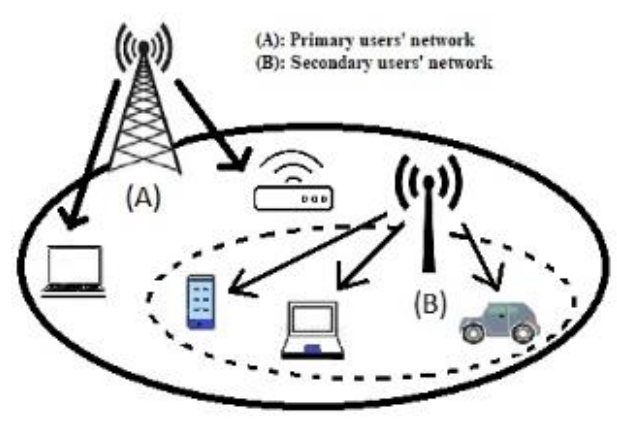

Figure 1. Cognitive radio system model
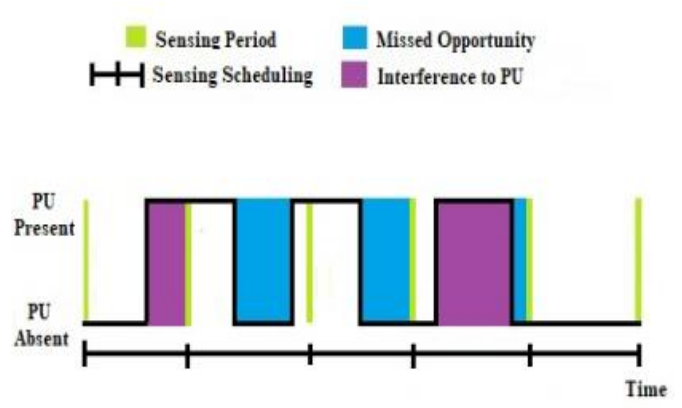

Figure 2. Spectrum sensing scheduling and its' effect

The sensing period may be defined as the time gap between two successive sensing events at a channel. Whereas, sensing time is defined as the time required for SU to sense the spectrum and detect the activities of PU. If the sensing period is either larger or shorter it harms the network. If the sensing period is chosen to be small, i.e. the SU senses the spectrum at a much higher rate. Then it may able to discover a large number of transmission opportunities and can respond to the return of PU much faster. Due to the smaller sensing period, SU detects PU's activities more accurately. This may reduce interference with PU. But as the system will be spending more time sensing the spectrum and will have much less time for data transmission. On the other hand, if the sensing period is made to be much larger it will allow the system sufficient time for data transmission but then the system will discover much lesser number of transmission opportunities as some of the transmission opportunities can be missed due to a large gap between consecutive sensing, as depicted in Figure 2. Larger sensing period may also be the reason for increased interference, as some activities of the PU may not be detected. Furthermore, the same sensing period cannot be made constant for all the channels as each channel has a particular usage pattern. The sensing period that yields good performance for one channel may not yield the same level of performance for another channel. That's why it is extremely important to optimize the sensing period for improvement of CR network's performance.

In this work, the PU's usage pattern of the spectrum is considered to be an ON-OFF model. We assume that $S(t)$ defines the channel state at any time instant t. $S(t)=0$, if the channel is available to the SU for transmission and $S(t)=1$, whenever the channel is unavailable to the SU. The sensing process is mainly the sampling of the channel state at regular intervals of time. To obtain the optimum set of sensing period for the channel, we use instantaneous sensing sample values as input to the GA.

GA is an optimizing technique based on the process of natural selection. It iterates through a defined set of solutions, called chromosomes, and evolves the solutions based on some function, known as the fitness function.

In this work, the number of opportunities incurred corresponding to each chromosome is evaluated, using the equation

$$
\varphi(x)=\sum_{i=1}^{n} \sum_{1}^{m} S\left(X_{i} \cdot k\right)
$$

The above (1), $\mathrm{X}_{\mathrm{i}}$ represents the randomly generated chromosome and $k$ varies between 1 to $\mathrm{m}$, which is the total number of sensing samples taken for the channel. The overhead cost for each chromosome is also calculated as the overhead must be within the desired limit. The overhead cost is considered as:

$$
\mathcal{C}(x)=\sum_{i=1}^{n} \frac{t_{s}}{X_{i}}
$$

Here, $t_{s}$ is the sensing time specified and is fixed.

The fitness for each chromosome is evaluated using the dominance and the threshold functions. The threshold function, in (4), is used to make sure that the overhead cost for the chromosome is under the desired limit and the dominance function given in (3) specifies the number of chromosomes fitness dominated by $\mathrm{j}^{\text {th }}$ chromosomes fitness, out of the total population [7]. 


$$
\begin{aligned}
& \mathcal{D}\left(X_{j}\right)=\sum_{r=1, r \neq j}^{l}\left\{\begin{array}{c}
1, X_{j \equiv} X_{r} \quad \text { (Dominance) } \\
0, X_{j \cong} X_{r}(\text { Non }- \text { dominance })
\end{array}\right. \\
& \Delta\left(X_{j}\right)=\left\{\begin{array}{l}
1, \mathcal{C}\left(X_{j}\right) \leq \text { Threshold } \\
0, \mathcal{C}\left(X_{j}\right)>\text { Threshold }
\end{array}\right.
\end{aligned}
$$

So the overall fitness function is given as,

$$
\xi\left(X_{j}\right)=\mathcal{D}\left(X_{j}\right)+\Delta\left(X_{j}\right)
$$

\section{SIMULATION AND RESULT ANALYSIS}

The parameters assumed for the GA is as follows: Initial population=100, Selection $=50 \%$, Mutation rate $=15 \%$, Number of bit/chromosome $=20$ bits. The population generated signifies sensing schedule. The population generated is binary numbers with 20 bits per chromosome. The parents for mating are chosen at random from the lower half of the sorted population. Each pair undergoes crossover. Single point crossover has been adopted in this work. Two offspring are obtained from each mating pair.

GA requires some stopping criteria to be defined. As soon as the stopping criterion is met, the GA stops iterating further. For this application the stopping criterion is defined as 100 iterations.

Figure 3 shows the spectrum occupancy of a licensed user for one hour. This is randomly generated by PU's transmission timing diagram where PU is transmitting with 0.25 probability. This spectrum occupancy pattern is considered to test the optimization method. For each chromosome, samples are taken from the signal. Each sample represents a specific sensing schedule. The state of the channel at that instant is determined from the signal. If the signal value at that instant is 1 then it signifies that the channel is occupied i.e. the PU is availing the channel and therefore there is no transmission opportunity for SU at that instant.

If the signal value is 0 for an instant, then it implies that the channel is free at that instant i.e the PU is currently not using the channel and the channel is idle. This presents a chance for SU to use the channel and carry out its communication. But, if the PU comes back for transmission before the next sensing takes place then this leads to interference with PUs. To minimize such interference this situation should be avoided. Therefore, while optimizing the sensing schedule only those samples are considered an opportunity to transmit, which are free of interference. It reduces the number of opportunities but it also reduces interference significantly.

Figure 4 shows the evolution of the maximum opportunities incurred as the function iterates through generations. The maximum opportunities incurred in the initial generations are very low. As the iterations increase, the chromosomes of the generations improve. By the process of mating the generations improves as the desired traits are carried on to the following generations but the undesired traits are eliminated.

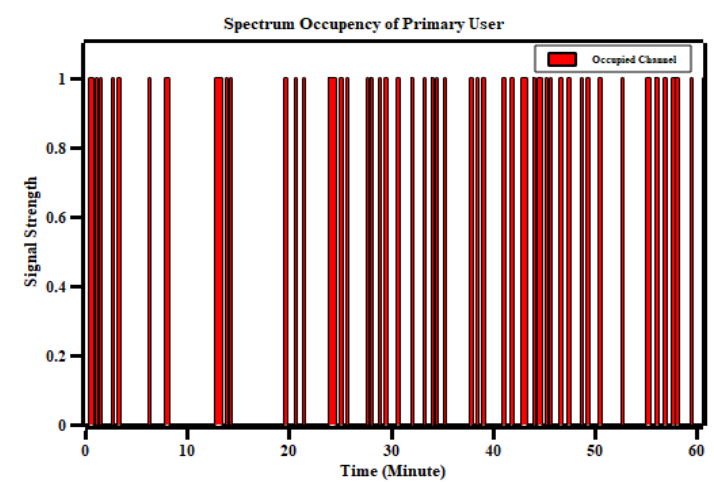

Figure 3. Spectrum occupancy of primary user

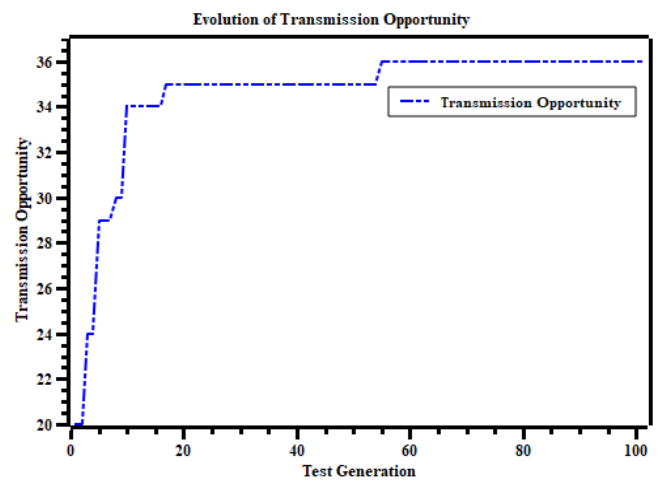

Figure 4. Transmission opportunity evolution with generations

While the GA iterates through generations, the performance of the fitness value initially is very poor but as the new generations are produced the fitness value gets better. Figure 5 shows the fluctuation observed in the average fitness through the iterations. It occurs as the processes of crossover and mutation not always produce a better offspring. Some of the offsprings have a worse fitness than their respective parent chromosomes. 
As mentioned earlier it is very essential to have the optimum value of the sensing period. Here the evolution of the sensing scheduling can be seen evidently. Over the generations, as the fitness value is calculated the corresponding sensing schedule is stored. The sensing schedule remains unchanged until a better sensing schedule is obtained, in this case, the previous sensing schedule is replaced by a new sensing schedule as the best sensing schedule. For the given spectrum usage pattern, an optimized sensing schedule is depicted in Figure 6.

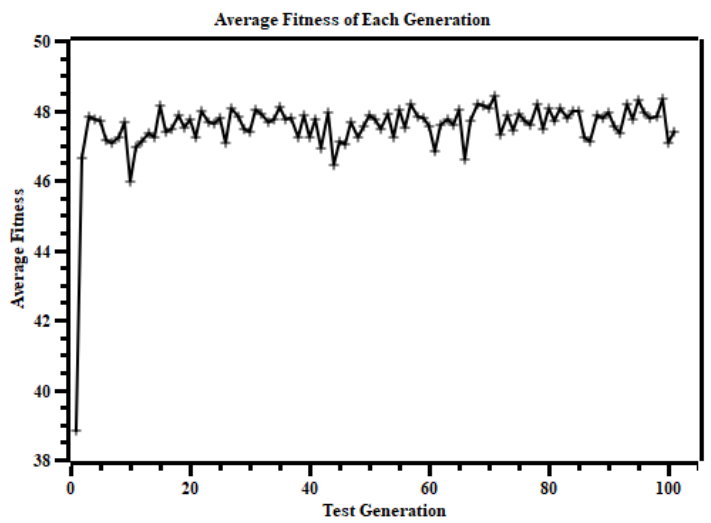

Figure 5. Average fitness of each generation

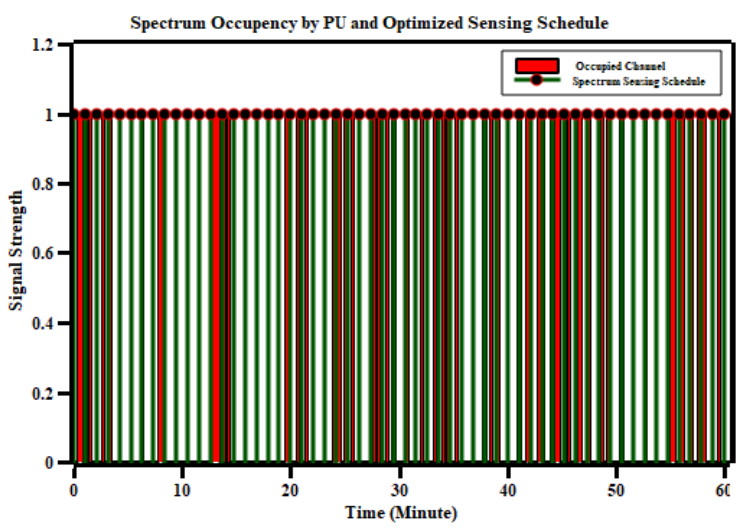

Figure 6. Optimized sensing schedule for given spectrum occupancy

\section{CONCLUSION}

As the numbers of wireless communicating devices are increasing, some new technologies must be introduced to increase the efficiency with which the spectrum is put to use. The major drawback of the present scenario of wireless communication is that a lot of frequencies are hardly used but as these are licensed to limited users they cannot be accessed by the users looking for a channel to communicate. In this state, opportunistic use of licensed spectrum which enables SUs to utilize the unused licensed band can be an effective solution to the problem. The concept of CR is based on the opportunistic use of PU's spectrum without interference to PU's communication.

This work shows that the optimized sensing schedule delivers the most number of non-interfering opportunities while keeping the overhead cost under constraint. It allows the CR to make optimum use of the available spectrum holes without interference or with minimum possible interference with the PU's communication. It presents a very effective way of using the spectrum as the proposed technique is not only maximizing the number of transmission opportunities but also minimizing interference with PU's transmission.

To improve upon this work, other optimization techniques can be used and results can be compared with the results of this work. As in modern communication, it is required that the decisions should be made by the hardware in the least amount of time, the comparison of various optimization techniques may help us in finding out the best-suited technique for this application in that regard as well.

\section{REFERENCES}

[1] Yucek, Tevfik and H. Arslan, "A survey of spectrum sensing algorithms for cognitive radio applications," IEEE communications surveys \& tutorials, pp. 116-130, 2009.

[2] M. I. J, "Cognitive radio: an integrated agent architecture for software defined radio," KTH, Stockholm, Sweden, 2000.

[3] F. C. Commission, "Spectrum policy task force report," FCC Document ET Docket No. 02-155., Nov 2002.

[4] S. Mansi and G. Birajdar, "Spectrum sensing techniques in cognitive radio networks: A survey," International Journal of Next-Generation Networks, vol. 3(2), pp. 37-51, 2011.

[5] S. Dasmahapatra, R. Sharma and S. N. Sharan, "A Review on Cognitive Radio: Smart Device for Next Generation Wireless Networks," INROADS-An International Journal of Jaipur National University, vol. 5(1s), pp. 120-124, 2016.

[6] G. Ganesan and Y. Li, "Cooperative spectrum sensing in cognitive radio, part II: multiuser networks," IEEE Transactions on wireless communications, vol. 6(6), pp. 2214-2222, 2007. 
[7] Balieiro and Andson, "A multi-objective genetic optimization for spectrum sensing in cognitive radio," Expert Systems with Applications, vol. 41.8, pp. 3640-3650, 2014.

[8] D. Niyato and H. Ekram, "Dynamics of network selection in heterogeneous wireless networks: An evolutionary game approach,” IEEE transactions on vehicular technology, vol. 58(4), pp. 2008-2017, 2009.

[9] J. Ren, "Dynamic channel access to improve energy efficiency in cognitive radio sensor networks," IEEE Transactions on Wireless Communications, vol. 15(5), pp. 3143-3156, 2016.

[10] S. Li, P. Gong, Q. Yang, M. G. Li and L. C. Ma, "Optimal spectrum sensing interval to minimize the transmission delay for cognitive radio," Advanced Materials Research, vol. 765, pp. 2657-2663, 2013.

[11] S. Chantaraskul and K. Moessner, "Implementation of a genetic algorithm-based decision making framework for opportunistic radio," IET communications, vol. 4, no. 5, pp. 495-506, 2010.

[12] N. A. Saoucha, A. C. Lammari and K. Benatchba, "Real-coded genetic algorithm parameter setting for cognitive radio adaptation," in International Conference on Smart Communications in Network Technologies (SaCoNeT), 2013.

[13] D. Bepari and D. Mitra, "Performance of GA in Power Allocation for Underlay Cognitive Radio Systems.," Journal of Communications Technology and Electronics, vol. 63, no. 10, pp. 1250-1258, 2018.

[14] K. V. Krishnan, R. M. Sajith and S. Khara, "Dynamic resource allocation in OFDM based cognitive radio system considering primary user QoS and secondary user proportional constraints," Journal of Communications Technology and Electronics, vol. 60, no. 11, pp. 1269-1275, 2015.

[15] S. DasMahapatra and S. N. Sharan, "A general framework for multiuser de-centralized cooperative spectrum sensing game," AEU-International Journal of Electronics and Communications, no. 92, pp. 74-81, 2018.

[16] S. DasMahapatra and S. N. Sharan, "Effect of Sensing Duration Optimization in Cooperative Spectrum Sensing Game," in 7th International Conference on Reliability, Infocom Technologies and Optimization (Trends and Future Directions)(ICRITO), IEEE, 2018.

[17] M. Kaushik, Y. Yoganandam and S. K.Sahoo, "Sensing and Sharing Schemes for Spectral Efficiency of Cognitive Radios," International Journal of Electrical and Computer Engineering (IJECE), vol. 8, no. 5, p. 2934 2941, October 2018.

[18] P. Varade, A. Wabale, R. Yerram and R. Jaiswal, "Throughput Maximization of Cognitive Radio Multi Relay Network with Interference Management," International Journal of Electrical and Computer Engineering (IJECE), vol. 8, no. 4, p. 2230 2238, August2018.

[19] S. D. Borde and K. R. Joshi, "Enhanced signal detection algorithm using trained neural network for cognitive radio receiver," International Journal of Electrical and Computer Engineering (IJECE), vol. 9, no. 1, p. 323 331, February 2019.

[20] S. E. Saad, I. F. Tarrad and A. A. Ammar, "Multi-Channel Preemptive Priority Model for Spectrum Mobility in Cognitive Radio Networks," International Journal of Electrical and Computer Engineering (IJECE), vol. 8, no. 6, p. 5169 5177, December2018.

[21] S Das Mahapatra, and S N Sharan. "Effect of Sensing Duration Optimization in Cooperative Spectrum Sensing Game." In 2018 7th International Conference on Reliability, Infocom Technologies and Optimization (Trends and Future Directions)(ICRITO), pp. 889-892. IEEE, 2018.

[22] Z. Zhenyu and X. Xie, "Intelligent cognitive radio: Research on learning and evaluation of CR based on neural network," in 5th international conference on Information and Communications Technology, 2007.

[23] Z. Zhenyu and X. Xie, "Application research of evolution in cognitive radio based on GA," in 3rd IEEE Conference on Industrial Electronics and Applications, 2008

[24] Z. Nan, S. Li and Z. Wu, "Cognitive radio engine design based on ant colony optimization," Wireless Personal Communications, vol. 65, no. 1, pp. 15-24, 2012.

[25] T. Newman and J. Evans, "Parameter sensitivity in cognitive radio adaptation engines," in 3rd IEEE Symposium on New Frontiers in Dynamic Spectrum Access Networks, 2008. 